\title{
TIROIDITIS AUTOINMUNE INDUCIDA POR INTERFERÓN EN PACIENTE CON INFECCIÓN POR VIRUS DE LA HEPATITIS C.
}

\author{
José L. Pinto1,3,a Miguel E. Pinto ${ }^{2,3, b}$
}

\begin{abstract}
RESUMEN
Se reporta el caso de un varón de 43 años de edad, sin antecedentes patológicos de importancia, que acudió por elevación asintomática de la alanino aminotransferasa (ALT). El paciente negó ser bebedor crónico de alcohol. Se hizo el diagnóstico serológico de infección activa por hepatitis $\mathrm{C}$ y la biopsia de hígado reveló inflamación crónica activa. Con estos resultados, se inició tratamiento con interferón-alfa y ribavirina. Durante el tratamiento de 48 semanas, el paciente presentó anticuerpos antitiroideos positivos con variaciones en sus niveles de tirotropina (TSH) y hormonas tiroideas. En el seguimiento postratamiento, el paciente continuó con hipertiroidismo por enfermedad de Graves. La tiroiditis autoinmune es una complicación frecuente del uso de interferón en pacientes con hepatitis $\mathrm{C}$. En algunos casos se presenta como hipertiroidismo por enfermedad de Graves. Se debe evaluar la función tiroidea y los anticuerpos antitiroideos antes y durante el tratamiento con interferón.
\end{abstract}

Palabras clave: Interferones; Hepatitis C; Tiroiditis; Enfermedad de Graves; Hipertiroidismo (fuente: DeCS BIREME).

\section{INTERFERON-INDUCED AUTOIMMUNE THYROIDITIS IN A PATIENT WITH HEPATITIS C VIRUS INFECTION}

\begin{abstract}
A 43 year old man presented with asymptomatic elevation of alanine aminotransferase (ALT) and no relevant past history. The patient denied being a chronic alcohol drinker. Work-up revealed an active hepatitis $\mathrm{C}$, and liver biopsy showed active inflammation. Treatment was started with interferon-alfa and ribavirin. During the 48 weeks of treatment, the patient developed positive thyroid antibodies with varying level of thyrotropin (TSH) and thyroid hormones. At follow-up after treatment, the patient continued with hyperthyroidism due to Graves' disease. Autoimmune thyroiditis is a common complication of using interferon in patients with hepatitis C. In some cases, it is presented as hyperthyroidism because of Graves' disease. Thyroid function and thyroid antibodies should be evaluated before and during treatment with interferon.
\end{abstract}

Keywords: Interferons; Hepatitis C; Thyroiditis; Graves Disease; Hyperthyroidism (source: MeSH NLM).

\section{INTRODUCCIÓN}

El tratamiento de la hepatitis $\mathrm{C}(\mathrm{HC})$ es la indicación más frecuente del interferón alfa (IFNa) ${ }^{(1)}$. En el $50 \%$ de los pacientes con infección crónica por $\mathrm{HC}$, el uso de peginterferón-alfa, reduce la carga viral a indetectable después de 24 semanas de tratamiento ${ }^{(2,3)}$. En el $40 \%$ de los pacientes, los eventos adversos asociados con el uso de IFNa obligan a reducir la dosis, mientras que en el $14 \%$, a suspender el tratamiento antiviral ${ }^{(4)}$. La tiroiditis inducida por IFNa (TII) es un evento adverso frecuente ${ }^{(4,5)}$ y ha sido clasificada como autoinmune o no autoinmune ${ }^{(6)}$.

En este reporte, describimos el caso de un paciente con $\mathrm{HC}$ activa que presentó TII. El paciente desarrolló anticuerpos antitiroideos (AAT) con variaciones en sus niveles de tirotropina (TSH) y hormonas tiroideas. El hipertiroidismo por enfermedad de Graves (EG) no remitió después de terminar el tratamiento antiviral.

\section{REPORTE DE CASO}

Se reporta el caso de un paciente varón de 43 años de edad, sin antecedentes de enfermedad tiroidea o hepática, quien acudió al consultorio de Gastroenterología por presentar elevación asintomática de la alanina aminotransferasa (ALT). El paciente negó ser bebedor crónico de alcohol. Se descartó la presencia de hepatitis autoinmune, infección por virus de la hepatitis B o por VIH. La ecografía hepática mostró leve esteatosis hepática, sin masas ni litiasis vesicular. Al persistir la elevación de la ALT, se solicitó evaluar anticuerpos antihepatitis C, los cuales fueron positivos. Posteriormente, se realizó un PCR cualitativo que confirmó la infección por el virus de la hepatitis $\mathrm{C}(\mathrm{VHC})$, genotipo 1a/1b. Dos meses después, se realizó una biopsia hepática, la cual confirmó el diagnóstico de hepatitis crónica activa, grado histológico II y estadio de fibrosis 2. La carga viral en ese momento fue de $850000 \mathrm{IU} / \mathrm{mL}$.

\footnotetext{
${ }^{1}$ Servicio de Gastroenterología, Hospital Nacional Cayetano Heredia, Lima, Perú.

2 Servicio de Endocrinología, Hospital Nacional Cayetano Heredia, Lima, Perú.

${ }^{3}$ Facultad de Medicina Alberto Hurtado, Universidad Peruana Cayetano Heredia, Lima, Perú.

${ }^{a}$ Médico gastroenterólogo; ${ }^{\mathrm{b}}$ Médico endocrinólogo.
} 
Se inició tratamiento con peginterferón-alfa-2a, $180 \mu \mathrm{g}$ SC por semana, y ribavirina $1200 \mathrm{mg}$ VO por día. Los controles pretratamiento de TSH y tiroxina libre (T4 libre) fueron normales (Tabla 1). En ese momento no se realizó la determinación de anticuerpos antiperoxidasa tiroidea (anti-TPO). Después de cuatro semanas de tratamiento, el paciente presentó mialgias, escalofríos, cefalea y diarrea. Los análisis de laboratorio mostraron elevación leve de TSH y reducción de la carga viral. El tratamiento no se modificó hasta la semana 12 , cuando se detectó TSH suprimido, T4 libre elevado, anemia, leucopenia y plaquetopenia. El peginterferón-alfa-2a fue reducido a $135 \mu \mathrm{g}$ SC por semana y la ribavirina a 1000 mg VO por día.

Después de 17 semanas de tratamiento, el TSH permaneció suprimido y el T4 libre elevado, sin embargo, no hubo síntomas de hipertiroidismo. En la semana 24 de tratamiento, la ALT se normalizó, la carga viral fue indetectable, pero el TSH se elevó a $26,15 \mathrm{mIU} / \mathrm{dL}$ (VN: 0,3 - 5) y el T4 libre disminuyó a 0,43 ng/dL (VN: 0,82). En ese momento, se midieron los anticuerpos antiTPO, los cuales fueron positivos. Se inició tratamiento con levotiroxina $50 \mu \mathrm{g}$ VO por día. El paciente completó 48 semanas de tratamiento sin presentar molestias. En el control final (semana 48), la ALT y el T4 libre fueron normales, sin embargo, el TSH nuevamente estuvo suprimido, pero no hubo síntomas de hipertiroidismo, por lo cual, se suspendió la levotiroxina.

En el control postratamiento (semana 72), la carga viral fue indetectable, la ALT permaneció normal, pero el TSH continuó suprimido. En ese momento, los niveles de anti- TPO se elevaron y los anticuerpos antitiroglobulina (anti Tg) se volvieron positivos. Con estos resultados, se confirmó el diagnóstico de EG como secuela de tiroiditis autoinmune inducida por el uso de peginterferón. El paciente ha permanecido asintomático, no ha recibido tratamiento para el hipertiroidismo y ha dejado de asistir a sus controles.

\section{DISCUSIÓN}

La hepatitis $\mathrm{C}$ es la causa más frecuente de enfermedad hepática crónica, cirrosis y hepatocarcinoma, con una prevalencia mundial del $3 \%{ }^{(7,8)}$. Varias enfermedades extrahepáticas han sido descritas en $\mathrm{HC}$, destacando las enfermedades hematológicas y los desórdenes autoinmunes ${ }^{(9,10)}$.

La disfunción tiroidea (DT) es frecuente en los pacientes con $\mathrm{HC}$, especialmente en mujeres. Los AAT pueden presentarse en $5-17 \%$ de las pacientes, y el hipotiroidismo primario, hasta en $2-13 \%$. La mayoría de los casos son asintomáticos y no requieren tratamiento ${ }^{(1,11)}$. En estos casos, el VHC podría desencadenar una respuesta autoinmune contra la glándula tiroides, especialmente, en personas con predisposición genética ${ }^{(5)}$. Una limitación de nuestro reporte es la falta de determinación de los AAT antes de iniciar el tratamiento con IFNa, sin embargo consideramos que el riesgo era bajo por ser el paciente un varón joven, sin antecedente de enfermedad tiroidea.

La tiroiditis es un evento adverso frecuente del IFNa en HC. Estudios previos han demostrado que hasta el 15\% de los pacientes tratados con IFNa desarrollan DT y hasta el $40 \%$, AAT ${ }^{(12)}$. Algunas de estas complicaciones pueden requerir la suspensión del tratamiento antiviral.

La TII puede ser clasificada en autoinmune o no autoinmune ${ }^{(6)}$. En el primer caso, puede manifestarse como EG, tiroiditis de Hashimoto $(\mathrm{TH})$ o presencia de AAT sin expresión clínica, mientras que en el segundo caso, la enfermedad se puede manifestar como una tiroiditis destructiva o un hipotiroidismo sin AAT ${ }^{(12)}$.

La TH es la manifestación clínica más frecuente de la TII de causa autoinmune ${ }^{(1)}$. El sexo femenino y la presencia de AAT antes del tratamiento con IFNa, son los factores de riesgo más importantes ${ }^{(12)}$. El desarrollo de EG es menos

Tabla 1. Resultados de laboratorio al diagnóstico y durante el seguimiento.

\begin{tabular}{|c|c|c|c|c|c|c|c|c|}
\hline & $\begin{array}{l}\text { Pretrata- } \\
\text { miento }\end{array}$ & $\begin{array}{c}\text { Semana } \\
4\end{array}$ & $\begin{array}{c}\text { Semana } \\
12\end{array}$ & $\begin{array}{c}\text { Semana } \\
17\end{array}$ & $\begin{array}{c}\text { Semana } \\
24\end{array}$ & $\begin{array}{c}\text { Semana } \\
34\end{array}$ & $\begin{array}{c}\text { Semana } \\
48\end{array}$ & $\begin{array}{c}\text { Semana } 72 \\
\text { postratamiento }\end{array}$ \\
\hline$\overline{A L T}(\mathrm{IU} / \mathrm{L})$ & 161 & - & - & - & 28 & - & 39 & 34 \\
\hline AST (IU/L) & 67 & - & - & - & - & - & - & \\
\hline $\mathrm{Hb}(\mathrm{g} / \mathrm{dL})$ & 17,0 & - & 12,7 & 13,5 & - & 11,6 & 12,2 & 16,6 \\
\hline Leucocitos & 5020 & - & 2570 & 3280 & - & - & 2880 & 6100 \\
\hline Plaquetas & 186000 & - & 145000 & - & - & - & - & - \\
\hline TSH $(\mu \mathrm{lU} / \mathrm{dL})$ & 2,37 & 4,99 & 0,023 & 0,012 & 26,15 & - & 0,08 & 0,005 \\
\hline T4 libre (ng/dL) & 0,99 & 1,27 & 3,28 & 4,3 & 0,43 & - & 1,46 & 1,85 \\
\hline Carga viral (IU/L) & 850000 & 23176 & - & - & 0 & - & - & 0 \\
\hline Anti TPO & - & - & - & - & 80 & 80 & - & 100 \\
\hline Anti Tg & - & - & - & - & 0 & 0 & - & 139 \\
\hline Levotiroxina ( $\mu \mathrm{g} / \mathrm{día})$ & - & - & - & - & 50 & 50 & - & - \\
\hline
\end{tabular}

$\mathrm{ALT}=$ alanino aminotransferasa (VN: 1-45); AST= aspartato aminotransferasa (VN: 1-36); Hb= hemoglobina (VN: 13-16 g/dL);

$\mathrm{TSH}=$ tirotropina (VN: 0.3-5); T4 libre= tiroxina libre (VN: 0.8-2); Anti-TPO= anticuerpos antiperoxidasa tiroidea $(\mathrm{VN}:<30 \mathrm{IU} / \mathrm{mL})$;

Anti- $\mathrm{Tg}=$ anticuerpos antitiroglobulina $(\mathrm{VN}:<30 \mathrm{IU} / \mathrm{mL})$ 
frecuente ${ }^{(6)}$, sin embargo, la posibilidad de remisión después de suspender el tratamiento con IFNa es mucho menor (1). En estos casos, hasta el $2 \%$ de los pacientes, van a presentar EG de manera permanente $(13,14)$. Por otro lado, la ribavirina es un nucleósido sintético con probables efectos inmunomodulares, que en asociación al IFNa puede desencadenar DT por un mecanismo autoinmune. La incidencia de DT en pacientes tratados con ribavirina en combinación con IFNa $(12,1 \%)$ es mayor a la que presentan los pacientes tratados solo con IFNa (6.6\%) ${ }^{(11)}$. En nuestro caso, el paciente permaneció con el TSH suprimido y con los AAT positivos después de 24 semanas de haber suspendido el tratamiento.

La tiroiditis destructiva (TD), representa el $50 \%$ de los casos de TII de causa no autoinmune ${ }^{(12)}$. Generalmente se presenta como un desorden inflamatorio, autolimitado, que se caracteriza por una fase tirotóxica seguida de una fase hipotiroidea, con recuperación total de la función hormonal en la mayoría de casos ${ }^{(1,6)}$. El proceso destructivo puede ser detectado de manera precoz con el uso de la ecografía ${ }^{(15)}$. En estos casos, menos del 5\% de los pacientes presentan hipotiroidismo permanente ${ }^{(16)}$. La patogénesis de la TII es desconocida, sin embargo, se plantean efectos tóxicos directos del IFNa sobre las células foliculares, especialmente, en los casos de TD ${ }^{(17)} \mathrm{y}$ efectos mediados por la inducción de autoinmunidad ${ }^{(18)}$.

En conclusión, la TII es una complicación frecuente del uso de IFNa. Los síntomas pueden ser confundidos con aquellos producidos por la $\mathrm{HC}$ o el uso de IFNa, por lo tanto, se recomienda un seguimiento cercano de la función tiroidea y los AAT.

\section{Contribuciones de Autoría}

Ambos autores participaron en la concepción, elaboración, revisión crítica y aprobación del documento final.

\section{Fuentes de Financiamiento}

Autofinanciado.

\section{Conflictos de Interés}

Los autores declaran no tener conflicto de interés en la publicación del presente artículo.

\section{REFERENCIAS BIBLIOGRÁFICAS}

1. Tomer $\mathbf{Y}$, Blackard JT, Akeno N. Interferon alpha treatment and thyroid dysfunction. Endocrinol Metab Clin North Am. 2007;36:1051-66.

2. Fried MW, Shiffman ML, Reddy KR, Smith C, Marinos G, Gonçales FL Jr, et al. Peginterferon alfa-2a plus ribavirin for chronic hepatitis $C$ virus infection. N Engl J Med. 2002;347:975-82.
3. Manns MP, McHutchinson JG, Gordon SC, Rustgi VK, Shiffman M, Reindollar R et al. Peginterferon alfa-2b plus ribavirin compared with interferon alfa-2b plus ribavirin for initial treatment of chronic hepatitis C: a randomised trial. Lancet. 2001;358:958-65.

4. Russo MW, Fried MW. Side effects of therapy for chronic hepatitis C. Gastroenterology. 2003;124:1711-19.

5. Carella C, Mazziotti G, Amato G, Braverman LE, Roti E. Interferon- $\alpha$ - related thyroid disease: Pathophysiological, epidemiological, and clinical aspects. J Clin Endocrinol Metab. 2004;89:3656-61.

6. Tomer $\mathbf{Y}$, Menconi F. Interferon induced thyroiditis. Best Pract Res Clin Endocrinol Metab. 2009;23:703-12.

7. Lauer GM, Walker BD. Hepatitis $\mathrm{C}$ virus infection. N Engl J Med. 2001;345:41-52.

8. Alter MJ. Epidemiology of hepatitis C virus infection. World J Gastroenterol. 2007;13:2436-41.

9. Killenberg PG. Extrahepatic manifestations of chronic hepatitis C. Semin Gastrointest Dis. 2000;11:62-8.

10. Sterling RK, Bralow S. Extrahepatic manifestations of hepatitis C virus. Curr Gastroenterol Rep. 2006;8:53-9.

11. Andrade LJ, Atta AM, D’Almeida Junior A, Paraná R. Thyroid dysfunction in hepatitis $C$ individuals treated with interferon-alpha and ribavirin-a review. Braz J Infect Dis. 2008;12:144-8.

12. Mandac JC, Chaudhry S, Sherman KE, Tomer $Y$. The clinical and physiological spectrum of interferon-alpha induced thyroiditis: toward a new classification. Hepatology. 2006;43:661-72.

13. Doi F, Kakizaki S, Takagi H, Murakami M, Sohara N, Otsuka $\mathrm{T}$, et al. Long-term outcome of interferon-alphainduced autoimmune thyroid disorders in chronic hepatitis C. Liver Int. 2005;25:242-6.

14. Kee KM, Lee CM, Wang JH, Tung HD, Changchien CS, Lu SN, et al. Thyroid dysfunction in patients with chronic hepatitis $C$ receiving a combined therapy of interferon and ribavirin: incidence, associated factors and prognosis. $J$ Gastroenterol Hepatol. 2006;21:319-26.

15. Friedrich-Rust $M$, Theobald J, Zeuzem S, Bojunga J. Thyroid function and changes in ultrasound morphology during antiviral therapy with pegylated interferon and ribavirin in patients with chronic hepatitis C. J Viral Hepat. 2009;16:168-77.

16. Prummel MF, Laurberg P. Interferon- $\alpha$ and autoimmune thyroid disease. Thyroid. 2003;13:547-51.

17. Tomer $\mathbf{Y}$. Hepatitis $C$ and interferon induced thyroiditis. J Autoimmun. 2010;34:J322-6.

18. Kong YM, Wei WZ, Tomer Y. Opportunistic autoimmune disorders. Ann N Y Acad Sci. 2010;1183:222-36.
Correspondencia: Miguel Pinto Valdivia

Dirección: Servicio de Endocrinología, Hospital Nacional Cayetano Heredia

Avenida Honorio Delgado 262, Lima 31, Perú

Teléfono: (511) 992-706110 / Fax: (511) 481-4177.

Correo electrónico: miguelpinto72@yahoo.com 